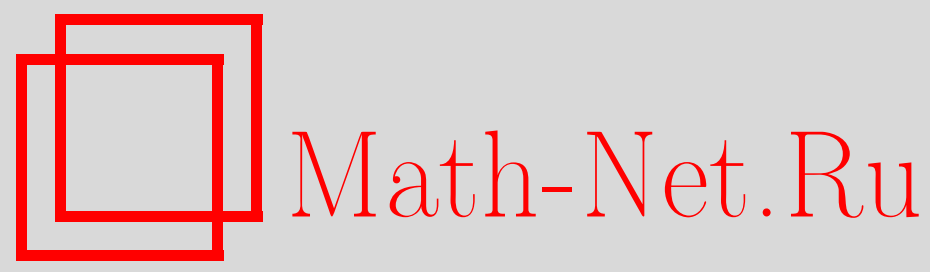

В. К. Белошапка, Симметрии вещественных гиперповерхностей трехмерного комплексного пространства, Матем. заметки, 2005, том 78, выпуск 2, 171-179

DOI: https://doi.org/10.4213/mzm2574

Использование Общероссийского математического портала Math-Net.Ru подразумевает, что вы прочитали и согласны с пользовательским соглашением http://www . mathnet.ru/rus/agreement

Параметры загрузки:

IP : 3.89 .197 .203

26 апреля 2023 г., 09:07:31

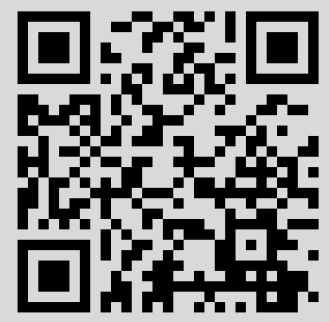




\title{
СИММЕТРИИ ВЕЩЕСТВЕННЫХ ГИПЕРПОВЕРХНОСТЕЙ ТРЕХМЕРНОГО КОМПЛЕКСНОГО ПРОСТРАНСТВА
}

\author{
В.К. Белошапка
}

\begin{abstract}
Основной результат работы - доказательство того, что для произвольного ростка вещественно аналитической гиперповерхности трехмерного комплексного пространства справедлива следующая альтернатива (гипотеза о размерности): либо размерность группы голоморфных симметрий ростка не превосходит размерности группы невырожденной гиперквадрики (она равна 15), либо эта группа бесконечномерна. Обсуждается также работа А. Ершовой, в которой обнаружены ошибки.
\end{abstract}

Библиографияя: 8 названий.

Риманова геометрия является образцом для многих дифференциально-геометрических построений и источником далеко идущих аналогий. В известной работе Черна и Мозера 1974 г. [1] проводилась параллель между римановой геометрией и геометрией вещественной гиперповерхности комплексного пространства (псевдоконформной геометрией в терминах Э. Картана). У этой аналогии имеется следующий аспект. В римановой геометрии известную роль играют однородные многообразия постоянной кривизны, так называемые модельные многообразия. Для этих во многих отношениях замечательных многообразий вьполнено, в частности, следуюшее экстремальное свойство. Они обладают максимальными по размерности группами симметрий. В геометрии вещественных подмногообразий комплексного пространства имеются вполне аналогичные объекты - модельные поверхности. Эти поверхности также (голоморфно) однородны, и группы их голоморфных симметрий имеют максимальную размерность.

Римановы многообразия можно в первом приближении различать по размерности; вещественные подмногообразия различают, прежде всего, по типу. Тип вещественного $d$-мерного порождающего подмногообразия $M^{d}$ комплексного пространства $\mathbb{C}^{N}$ - это пара чисел $(n, K)$, где $n$ - комплексная размерность комплексного касательного пространства (CR-размерность), а $K$ - вещественная коразмерность, при этом $K+n=N$ и $K+2 n=d$. Например, гиперповерхность в пространстве $\mathbb{C}^{n+1}$ имеет тип $(n, 1)$. В этой ситуации модельной поверхностью является гиперквадрика вида $\operatorname{Im} w=\langle z, \bar{z}\rangle$, где $\langle z, \bar{z}\rangle$ - невырож денная эрмитова форма от $n$-мерной переменной $z$. Если зафиксировать значение $n$ и начать увеличивать $K$, то пока $K \leqslant n^{2}$, модельные поверхности будут

Работа выполнена при финансовой поддержке Российского фонда фундаментальных исследований, гранты № 0015-96-008 и № 02-01-01-291. 
задаваться системой вещественно квадратичных уравнений, затем степени определяющих уравнений начнут возрастать [2]. Недавно задача построения модельной поверхности была решена для многообразий произвольного типа [3]. Среди свойств модельной поверхности имеется следующее: размерность локальной группы голоморфных автоморфизмов произвольного вполне невырожденного ростка не превосходит размерности группы касательной модельной поверхности.

Если условие полной невырожденности отбросить, то группа может стать бесконечномерной. Однако можно сформулировать следующую гипотезу, контрпример к которой нам неизвестен.

ГипотЕЗА. Для размерности локальной групшы автоморфизмов произвольного вещественно аналитического ростка имеет место следующая альтернатива. Либо эта размерность равна бесконечности, либо она не превосходит размерности группы модельной поверхности соответствуюшего типа. То есть максимум размерности групш голоморфных симметрий (в классе конечномерных груп) достигается не на вырожденных ростках, а на невырожденных модельных поверхностях.

Эта гипотеза доказана для многообразий типа $(1,1)[2]$ и $(1,2)[4]$. В [2] автором было приведено рассуждение, доказьвающее эту гипотезу для гиперповерхности в $\mathbb{C}^{3}$, т.е. для многообразий типа $(2,1)$. Это рассуждение существенно использовало рузультаты работы А. Ершовой [5], которая дала оценки размерности групшы автоморфизмов для каждого из восьми возможных типов 2-невырожденных гиперповерхностей, описанных П. Эбенфельтом [6]. В работе Ершовой содержится утверждение, что размерность локальной группы автоморфизмов 2-невырожденного ростка не превосходит 7. Однако Д. Зайцев обратил мое внимание на то, что размерность группы голоморфных симметрий “светового" конуса $(\operatorname{Im} w)^{2}=(\operatorname{Im} z)^{2}+(\operatorname{Im} \zeta)^{2}$ равна $10($ см. [7], а также обсуждение в конце данной работы). Этот пример не опровергает нашей гипотезы о размерности, так как размерности груп обеих невырожденных гиперквадрик, положительно определенной $\operatorname{Im} w=|z|^{2}+|\zeta|^{2}$ и знаконеопределенной $\operatorname{Im} w=|z|^{2}-|\zeta|^{2}$, равны 15, но он находится в противоречии с оценкой Ершовой.

В данной работе дается независимое от результатов Ершовой доказательство гипотезы для гиперповерхностей в $\mathbb{C}^{3}$ и проводится анализ ее работы.

Для дальнейшего нам понадобятся следуюшие обозначения. Если $M-$ гладкая поверхность в $\mathbb{C}^{N}, \xi$ - ее точка, $M_{\xi}$ - росток $M$ в точке $\xi$, то через aut $M_{\xi}$ обозначим алгебру Ли ростков вещественных векторных полей с голоморфньми коэффициентами в точке $\xi$, касательных к $M_{\xi}$. Если $z=\left(z_{1}, \ldots, z_{N}\right)$ - координаты в $\mathbb{C}^{N}$, то

$$
\text { aut } M_{\xi}=\left\{X(z)=2 \operatorname{Re}\left(f_{1}(z) \frac{\partial}{\partial z_{1}}+\cdots+f_{N}(z) \frac{\partial}{\partial z_{N}}\right)\right\}
$$

где сужение поля $X$ на $M_{\xi}$ есть росток поля, касательного к $M_{\xi}$, а коэффициенты $\left(f_{1}(z)\right.$, $\left.\ldots, f_{n}(z)\right)$ - ростки функций, голоморфных в $\xi$. Это алгебра Ли инфинитезимальных голоморфных автоморфизмов, т.е. векторных полей, которые порождают голоморфное действие на $M_{\xi}$. В ней имеется подалгебра aut $M_{\xi}$, состоящая из ростков полей, обращающихся в нуль в точке $\xi$. Можно рассмотреть соответствующую aut $M_{\xi}$ локальную группу Aut $M_{\xi}$ и соответствующую aut ${ }_{\xi} M_{\xi}$ группу Aut ${ }_{\xi} M_{\xi}$, т.e. Aut $M_{\xi}$ и $\operatorname{Aut}_{\xi} M_{\xi}-$ 
это образы aut $M_{\xi}$ и aut $M_{\xi}$ под действием экспоненциального отображения. Эти группы действуют на $M_{\xi}$ отображениями, биголоморфными в $\xi$. Причем Aut $M_{\xi}-$ это стабилизатор точки $\xi$ в Aut $M_{\xi}$, т.е. эта подгруппа состоит из тех преобразований из $\mathrm{Aut}_{\xi} M_{\xi}$, которые сохраняют точку $\xi$ неподвижной.

Приведем определение $q$-невырожденности из работы [6]. Пусть $\rho(Z, \bar{Z})=0$ - локальное уравнение $M$ в окрестности $\xi$ и существует положительное целое число $l$ такое, что $\operatorname{span}\left\{L^{\alpha} \rho_{Z}(\xi, \bar{\xi}):|\alpha| \leqslant l\right\}=\mathbb{C}^{N}$, где $L^{\alpha}=L_{1}^{\alpha_{1}} \cdots L_{n}^{\alpha_{n}}$, причем $L_{1}, \ldots, L_{n}-$ базис CR-векторных полей на $M$ в окрестности $\xi$, а $\rho_{Z}=\partial \rho / \partial Z$ - голоморфньй градиент. Тогда говорим, что $M q$-невыр ождена в точке $\xi$, если $q$-минимальноеиз таких чисел $l$.

Пусть $\Gamma$ - вещественно аналитическая гиперповерхность в $\mathbb{C}^{3}$ с координатами $(z, \zeta$, $w=u+i v)$, тогда имеется три взаимоисключающие возможности. Либо в точке общего положения (т.е. на открытом плотном подмножестве) форма Леви Г имеет ранг 2 (т.е. невырождена), либо в точке общего положения она имеет ранг 1 , либо ранг 0. В первом случае мы сразу получаем требуемую оценку [1]. Третий случай - это Леви-плоская гиперповерхность, она локально эквивалентна гиперплоскости, и ее групп бесконечномерна. Интерес представляет второй случай. Такая гиперповерхность в точке общего положения должна быть, к тому же, 2-невырожденной, и ее локальное уравнение может быть записано в одной из трех форм [6]:

(I) $v=|z|^{2}+|\zeta|^{2}(\zeta+\bar{\zeta})+\gamma\left(z^{2} \bar{\zeta}+\bar{z}^{2} \zeta\right)+O\left(|z|^{4}+|u||z|^{2}\right)$, где $\gamma=0,1$;

(II) $v=|z|^{2}+\left(z^{2} \bar{\zeta}+\bar{z}^{2} \zeta\right)+O\left(|z|^{4}+|u||z|^{2}\right)$;

(III) $v=|z|^{2}+|\zeta|^{2}(z+\bar{z})+O\left(|z|^{4}+|u||z|^{2}\right)$.

Оценки работы [5] основаны на применении следующей, многократно применявшейся ранее конструкции. Введем в пространстве рядов от переменных $(z, \bar{z}, w, \bar{w})$ градуировку, назначая некоторым образом положительные веса переменным. Обозначим через $O(q)$ выражение, являющееся суммой компонент веса $q$ и выше. Тогда размерность семейства отображений, переводящих росток вида

$$
v=F_{m}+O(m+1)
$$

в другой росток такого же вида $v=\widetilde{F}_{m}+O(m+1)$, где $F_{m}$ и $\widetilde{F}_{m}$ - однородные многочлены веса $m$, и сохраняющих начало координат, не превосходит размерности алгебры Ли aut $Q_{0}$ гиперповерхности $Q=\left\{v=F_{m}(z, \bar{z}, u)\right\}$. Это доказывается рекуррентным вычислением и представляет собой версию теоремы о неявной функции [1], [2].

Если положить $[z]=3,[\zeta]=2,[w]=6$, то поверхность вида (I) записьвается как $v=F_{6}+O(7)$, поэтому размерность групш ростка оценивается размерностью алгебры гиперповерхности $Q^{1}=\left\{v=|z|^{2}+|\zeta|^{2}(\zeta+\bar{\zeta})\right\}$.

Продолжим введенную градуировку на векторные поля, полагая

$$
\left[\frac{\partial}{\partial z}\right]=-3, \quad\left[\frac{\partial}{\partial \zeta}\right]=-2, \quad\left[\frac{\partial}{\partial w}\right]=-6 .
$$

В этой градуировке все векторные поля распадаются на весовые компоненты от минус шестой и выше. В силу весовой однородности уравнения $Q^{1}$ алгебра инфинитезимальных автоморфизмов этой гиперповерхности обладает следующим свойством. Вместе с векторньм полем эта алгебра содержит каждую его весовую компоненту.

Используя стандартную технику работы со степенньми рядами, получаем описание алгебры инфинитезимальных автоморфизмов гиперповерхности $Q^{1}$. 
ПРЕДЛОЖЕНИЕ 1. Если $\Gamma_{0}^{1}-$ росток 2-невырожсднной гиперповерхности в нуле muna (I), a $Q^{1}=\left\{v=|z|^{2}+|\zeta|^{2}(\zeta+\bar{\zeta})\right\}$, mo

(а) алгебра aut $Q^{1}$ имеет следующую градуированную структуру:

$$
g_{-6}+g_{-3}+g_{-2}+g_{0}
$$

при этом

$$
\begin{gathered}
g_{-6}=\left\{2 \operatorname{Re}\left(a \frac{\partial}{\partial w}\right)\right\}, \quad g_{-3}=\left\{2 \operatorname{Re}\left(2 i \bar{b} z \frac{\partial}{\partial w}+b \frac{\partial}{\partial z}\right)\right\} \\
g_{-2}=\left\{2 \operatorname{Re}\left(2 c \zeta^{2} \frac{\partial}{\partial w}+i c \frac{\partial}{\partial \zeta}\right)\right\} \\
g_{0}=\left\{2 \operatorname{Re}\left(6 d w \frac{\partial}{\partial w}+3(d+i e) z \frac{\partial}{\partial z}+2 d \zeta \frac{\partial}{\partial \zeta}\right)\right\}
\end{gathered}
$$

где $a, c, d, e \in \mathbb{R}, b \in \mathbb{C}$, в частности, размерности аut $Q^{1} u$ aut $_{0} Q^{1}=g_{0}$ равны 6 и 2 соответственно;

(b) выполнены оценки

$$
\operatorname{dim} \mathrm{Aut}_{0} \Gamma_{0}^{1} \leqslant 2, \quad \operatorname{dim} \mathrm{Aut} \Gamma_{0}^{1} \leqslant 6 .
$$

Второе неравенство в (b) следует из (a), первого неравенства (b) и того, что росток этого типа не может быть однороден [6], т.е. коразмерность $\operatorname{aut}_{0} Q^{1}$ в aut $Q^{1}$ не больше 4 . Заметим, что $Q^{1}$ реализует максимум размерности и полной группы для таких ростков, так как $\operatorname{dim}$ Aut $Q^{1}=6$.

Полученный мною ответ не совпадает с тем, что дан в статье Ершовой. Если разложить полученньй ею ответ по весовым компонентам, то алгебра получает следующую структуру: $g_{-6}+g_{-3}+g_{-2}+g_{0}+g_{6}$, где все компоненты, за исключением последней, совпадают с нашими. При этом, нетрудно проверить, что поле

$$
2 \operatorname{Re}\left(z w \frac{\partial}{\partial z}+w^{2} \frac{\partial}{\partial w}\right)
$$

порождающее ее компоненту $g_{6}$, не является касательным к $Q^{1}$.

Переходим к рассмотрению поверхностей вида (III). Если теперь положить $[z]=2$, $[\zeta]=1,[w]=4$, то такая поверхность записьвается как $v=F_{4}+O(5)$, поэтому размерность групшы ростка оценивается размерностью алгебры aut $_{0} Q^{3}$ гиперповерхности $Q^{3}=\left\{v=|z|^{2}+|\zeta|^{2}(z+\bar{z})\right\}$. Проводя стандартные вычисления со степенньги рядами, получаем описание алгебры инфинитезимальных автоморфизмов гиперповерхности $Q^{3}$.

ПРЕДЛОЖЕНИЕ 2. Если $\Gamma_{0}^{3}-$ росток 2-невырожденной гиперповерхности в нуле muna (III), a $Q^{3}=\left\{v=|z|^{2}+|\zeta|^{2}(z+\bar{z})\right\}$, mo

(а) алгебра aut $Q^{3}$ имеет следующую градуированную структуру:

$$
g_{-4}+g_{-2}+g_{0}
$$


при этом

$$
\begin{gathered}
g_{-4}=\left\{2 \operatorname{Re}\left(a \frac{\partial}{\partial w}\right)\right\}, \quad g_{-2}=\left\{2 \operatorname{Re}\left(2 i b z \frac{\partial}{\partial w}+i b \frac{\partial}{\partial z}\right)\right\} \\
g_{0}=\left\{2 \operatorname{Re}\left(4 c w \frac{\partial}{\partial w}+2 c z \frac{\partial}{\partial z}+(c+i d) \zeta \frac{\partial}{\partial \zeta}\right)\right\}
\end{gathered}
$$

әде $a, b, c, d \in \mathbb{R}$, в частности, размерности аut $Q^{3} u$ aut $_{0} Q^{3}=g_{0}$ равнь 4 и 2 соответственно;

(b) выполнены оченки

$$
\operatorname{dim} \mathrm{Aut}_{0} \Gamma_{0}^{3} \leqslant 2, \quad \operatorname{dim} \mathrm{Aut} \Gamma_{0}^{3} \leqslant 6 .
$$

Второе неравенство в (b), как и в предыдущем случае, следует из (a), первого неравенства (b) и того, что росток этого типа не может быть однороден [6], т.е. коразмерность aut $_{0} Q^{1}$ в aut $Q^{1}$ не больше 4 . Однако в этом случае, мы не можем утверждать, что оценка размерности полной группы ростка этого вида точна. Как и в предыдущем случае, полученньй мною ответ не совпадает с тем, что дан в статье Ершовой. Если разложить полученньй ею ответ по весовым компонентам, то алгебра получает следующую структуру: $g_{-4}+g_{-2}+g_{0}+g_{2}$, где все компоненты, за исключением последней, совпадают с нашими. При этом, нетрудно проверить, что поле

$$
2 \operatorname{Re}\left(\left(2 i z^{2}+w\right) \frac{\partial}{\partial z}+2 i z w \frac{\partial}{\partial w}\right)
$$

порождающее ее компоненту $g_{2}$, не является касательным к $Q^{3}$.

Теперь, для доказательства сформулированной гипотезы нам нужна оценка размерности стабилизатора точки для гиперповерхностей вида (II). Своеобразие этого вида заключается в следующем. Не существует выбора весов переменных $z$ и $\zeta$ такого, чтобы многочлен $|z|^{2}+\left(z^{2} \bar{\zeta}+\bar{z}^{2} \zeta\right)$ стал однородным, а остальные члены получили более высокий вес. Таким образом, оценка размерности групш гиперповерхности $Q^{2}=\left\{v=|z|^{2}+\left(z^{2} \bar{\zeta}+\bar{z}^{2} \zeta\right)\right\}$ (Ершова получила правильные ответы: 3 - размерность стабилизатора и 6 - полная размерность) ничего неговорит о размерности группы ростка этого вида. В этом главная ошибка работы Ершовой.

В классификации Эбенфельта имеется еще пять видов ростков. Это 2-невырожденные ростки, чья форма Леви обращается в нуль в центре ростка. Эти ростки в точке общего положения обязаны иметь форму Леви ранга не ниже 1 , и поэтому для получения оценки на размерность группы нам нет нужды рассматривать их отдельно. Однако каждьй из этих пяти ростков допускает при правильной расстановке весов запись в виде (1). Поэтому можно утверждать, что размерности подалгебр соответствующих модельных поверхностей мажорируют размерности стабилизаторов точки в группах ростков.

Вернемся к обсуждению ростков вида (II). Чтобы получить оценку можно, конечно, поступить так. Можно положить $[z]=[\zeta]=1,[w]=2$, и тогда уравнение запишется в виде

$$
v=|z|^{2}+O(3)
$$


и можно будет оценивать размерность стабилизатора нуля через размерность стабилизатора нуля гиперповерхности $v=|z|^{2}$; но беда в том, что она равна бесконечности. Второй вариант - это двухшаговая редукция. Сначала надо ввести веса, как это сделано вьше, записать поверхность в виде (2) и явно описать функциональные параметры из бесконечномерной алгебры Ли поверхности $v=|z|^{2}$, которые будут однозначно определять отображение ростка вида (2) в другой такой же росток. А затем, на втором шаге, надо воспользоваться тем, что компонента уравнения веса 3 отлична от нуля (несферичность) и имеет известный вид $F_{3}=\left(z^{2} \bar{\zeta}+\bar{z}^{2} \zeta\right)$, и получить новые ограничения на эти параметры.

Однако есть еще одна возможность, которой мы и воспользуемся. Можно процесс рекуррентного вычисления строить с привязкой не к самому младшему члену уравнения гиперповерхности, а к двум младшим, в данном случае - квадратичному и кубическому.

Итак, задаем градуировку, назначая $[z]=[\zeta]=1,[w]=2$. Пусть имееются две гиперповерхности

$$
\begin{aligned}
& v=|z|^{2}+\left(z^{2} \bar{\zeta}+\bar{z}^{2} \zeta\right)+\sum_{4}^{\infty} F_{j}(z, \bar{z}, \zeta, \bar{\zeta}, u), \\
& v=|z|^{2}+\left(z^{2} \bar{\zeta}+\bar{z}^{2} \zeta\right)+\sum_{4}^{\infty} \widetilde{F}_{j}(z, \bar{z}, \zeta, \bar{\zeta}, u)
\end{aligned}
$$

и отображение первой во вторую вида

$$
z \rightarrow z+\sum_{3}^{\infty} f_{j}(z, \zeta, w), \quad \zeta \rightarrow \zeta+\sum_{2}^{\infty} g_{j}(z, \zeta, w), \quad w \rightarrow w+\sum_{4}^{\infty} h_{j}(z, \zeta, w) .
$$

Если координату $w$ в отображении (4) ограничить на первую гиперповерхность, то его значения будут удовлетворять уравнению второй гиперповерхности, и мы получаем некоторое соотношение в степенных рядах. Введем обозначения $w_{2}=u+i|z|^{2}$, $w_{3}=2 i \operatorname{Re}\left(z^{2} \bar{\zeta}+\bar{z}^{2} \zeta\right)$. Определим линейньй оператор вида

$$
\begin{aligned}
L(f, g, h) & =-\operatorname{Im} h\left(z, \zeta, w_{2}\right)+2 \operatorname{Re}\left(f\left(z, \zeta, w_{2}\right) \bar{z}+g\left(z, \zeta, w_{2}\right) \bar{z}^{2}\right) \\
D L(f, g, h) & =-\operatorname{Im}\left(h_{w}^{\prime}\left(z, \zeta, w_{2}\right) w_{3}\right)+2 \operatorname{Re}\left(f_{w}^{\prime}\left(z, \zeta, w_{2}\right) \bar{z} w_{3}+g_{w}^{\prime}\left(z, \zeta, w_{2}\right) \bar{z}^{2} w_{3}\right) .
\end{aligned}
$$

Тогда $m$-я компонента нашего соотношения имеет вид

$$
L\left(f_{m-1}, g_{m-2}, h_{m}\right)+D L\left(f_{m-2}, g_{m-3}, h_{m-1}\right)=\cdots
$$

где точками обозначено выражение, зависящее лишь от компонент отображения меньших весов. Поэтому можно утверждать, что размерность множества решений (число свободных параметров) в классе отображений вида (4) не превосходит размерности линейного пространства решений однородной системы

$$
\mathscr{L}(f, g, h)=L(f, g, h)+D L(f, g, h)=0 .
$$

Вычислим ядро опрератора $\mathscr{L}$. 
ПРЕДЛОЖЕНИЕ 3. Решение этого однородного линейного уравнения одномерно и имеет следующий вид:

$\operatorname{Ker} \mathscr{L}=\{f=0, g=0, h=c\}$

әде с-вешественная постоянная.

ДокАЗАтЕЛЬСтво. Полагая в $(5) \bar{z}=0$, видим, что $h$ не зависит от $\zeta$. Дифференцируя это соотношение по $\bar{z}$ и полагая $\bar{z}=0$, видим, что $f$ не зависит от $\zeta$. Дифференцируя его по $\bar{z}$ дважды и полагая $\bar{z}=0$, видим, что и $g$ не зависит от $\zeta$. Теперь соотношение можно записать так:

$$
2 \operatorname{Re}\left(T\left(z, \bar{z}, w_{2}\right)+i\left(z^{2} \bar{\zeta}+\bar{z}^{2} \zeta\right) T_{w}^{\prime}\left(z, \bar{z}, w_{2}\right)\right)=0
$$

где $T=i h+2 f \bar{z}+2 g \bar{z}^{2}$. Это после дифференцирования по $\zeta$ дает $\operatorname{Re} T_{w}^{\prime}=0$, откуда получаем, что $T_{w}^{\prime}=0$ и, следовательно, $f, g, h$ не зависят от $w$. Теперь уравнение принимает вид $\operatorname{Re} T=0$, откуда и получаем окончательное описание ядра.

Рассмотрим произвольное локально обратимое голоморфное отображение между ростками (3), сохраняющее начало координат на месте:

$$
z \rightarrow \sum_{1}^{\infty} f_{j}(z, \zeta, w), \quad \zeta \rightarrow \sum_{1}^{\infty} g_{j}(z, \zeta, w), \quad w \rightarrow \sum_{1}^{\infty} h_{j}(z, \zeta, w) .
$$

Анализ младших компонент возникающего при этом соотношения показьвает, что имеет место следующее утверждение.

ПРЕДЛОЖЕНИЕ 4. (а) Преобразование

$$
z \rightarrow \lambda z, \quad \zeta \rightarrow \lambda(\bar{\lambda})^{-1} \zeta, \quad w \rightarrow \lambda \bar{\lambda} w
$$

әде $\lambda$ - ненулевое комплексное число, является автоморфизмом гиперповерхности $Q^{2}$ и сохраняет вид ростка (3).

(b) Любое преобразование, сохраняющее вид уравнения (3), такое, что $f_{z}^{\prime}(0,0,0)$ $=1$, имеет вид

$z \rightarrow z+a w+(2 i \bar{a}-\bar{\mu}) z^{2}+O(3), \quad \zeta \rightarrow \mu z+\zeta+O(2), \quad w \rightarrow w+2 i \bar{a} z w+O(4)$,

где а и

(с) Любое обратимое преобразование, сохраняющее вид уравнения (3) и начало координат, можно однозначно представить в виде композичии преобразований вида (a) и (b) из данного утверждения, а затем вида (4).

В итоге мы получаем следующее

ПРЕДЛОЖЕНИЕ 5. Размерность подгруппы автоморфизмов ростка гиперповерхности вида (II), сохраняющих точку, не превосходит 6.

Размерность всей локальной группы автоморфизмов гиперповерхности вида (II) не превосходит $5+6=11$.

И это позволяет сформулировать окончательный результат, которьй доказывает нашу гипотезу для $\mathbb{C}^{3}$ в следующей уточненной форме. 
ТЕорема 6. Если росток вещественно аналитической гиперповерхности в $\mathbb{C}^{3}$ не является ни Леви-плоским (размерность группы равна бесконечности), ни сферическим (размерность равна 15), то размерность его локальной группь автоморфизмов не превосходит 11. Если же известно, что росток в общей точке не является ростком вида (II), то размерность әруппы не превосходит 6.

Световой конус

$$
S=\left\{(\operatorname{Im} w)^{2}=(\operatorname{Im} z)^{2}+(\operatorname{Im} \zeta)^{2}\right\},
$$

который послужил поводом для данной работы, - это трубчатая поверхность над квадратичным конусом в $\mathbb{R}^{3}$. Можно показать, что локальные автоморфизмы этой гиперповерхности продолжаются до глобальных и, в частности, в окрестность острия. Если задать градуировку полей условиями $[z]=[\zeta]=[w]=1$, то видно, что алгебра конуса aut $S_{0}$ имеет следующую градуированную структуру: $g_{-1}+g_{0}+g_{1}$. При этом

$$
g_{-1}=\left\{2 \operatorname{Re}\left(a_{1} \frac{\partial}{\partial z}+a_{2} \frac{\partial}{\partial \zeta}+a_{3} \frac{\partial}{\partial w}\right)\right\},
$$

где $a \in \mathbb{R}^{3}$. Эта подалгебра соответствует группе вещественных параллельных переносов. Далее, $g_{0}$ - это подалгебра, соответствующая подгруппе линейных преобразований $S$, т.е. подгрупше конформных преобразований, порожденньг вещественньпи преобразованиями, сохраняющими квадратичную форму $-y_{1}^{2}-y_{2}^{2}+y_{3}^{2}$, и вещественньми скалярньми растяжениями. Подалгебра $g_{1}$ изоморфно отображается на $g_{-1}$ при инверсии $(z, \zeta, w) \mapsto(z, \zeta, w) /\left(-z^{2}-\zeta^{2}+w^{2}\right)$. Соответствующая подгруппа порождается инверсией и переносами, т.е. состоит из инверсий с центрами в произвольных точках острия. Итого, размерность группы действительно равна $3+(3+1)+3=10$.

Конус в $\mathbb{R}^{3}$ - это линейчатая поверхность (1-параметрическое семейство вещественных прямых), а наша трубка в $\mathbb{C}^{3}$ - это семейство комплексных прямых, параметризованное тремя вещественными параметрами. То, что через каждую точку $S$ проходит комплексная прямая, позволяет заключить, что форма Леви имеет ранг не вьше единицы. Если бы этот ранг был равен нулю, то гиперповерхность была бы локально плоской, т.е. локально голоморфно эквивалентной вещественной гиперплоскости, и тогда наше 3-параметрическое семейство комплексных прямых было бы локально голоморфно эквивалентно 1-параметрическому семейству 2-мерных комплексных плоскостей, т.е. было бы распрямляемо, а группа автоморфизмов была бы бесконечномерной. Но это не так. Группа конечномерна, семейство не распрямляемо, а форма имеет ранг 1 [7].

В заключение два вопроса.

Полученная оценка - 11 - на единицу отличается от имеющегося примера с десятимерной группой. Этот досадный зазор оставляет открытьм вопрос о точной оценке, в отличие от оценки на размерность групп автоморфизмов гиперповерхностей, которые в общей точке не принадлежат виду (II). Как было показано выше, для них оценка 6 является точной.

И второй вопрос. Является ли световой конус единственной локально однородной гиперповерхностью в $\mathbb{C}^{3}$ с формой ранга один или же есть и другие? Как известно [8], локально однородных невырожденных гиперповерхностей в $\mathbb{C}^{3}$ очень много, как положительно определенных, так и знаконеопределенных. С другой стороны, единственной однородной гиперповерхностью с формой ранга нуль является гиперплоскость. 


\section{СПИСОК ЦИТИРОВАННОЙ ЛИТЕРАТУРЫ}

[1] Chern S.S., Moser J.K. Real hypersurfaces in complex manifold // Acta Math. 1974. V. 133. №3-4. P. 219-271.

[2] Белошапка В. К. Вещественные подмногообразия комплексного пространства их полиномиальные модели, автоморфизмы и проблемы классификации // УМН. 2002. Т. 57. №1. C. 3-44.

[3] Белошапка В.К. Универсальная модель вещественного подмногообразия // Матем. заметки. 2004. Т. 75. № 4. С. 507-522.

[4] Белошапка В. К., Ежов В. В., Шмальц Г. Голоморфная классификация четырехмерных поверхностей в $\mathbb{C}^{3} / /$ Изв. РАН. Сер. матем. (в печати).

[5] Ершова А. Е. Автоморфизмы 2-невырожденных гиперповерхностей в $\mathbb{C}^{3} / /$ Матем. заметки. 2001. T. 69. № 2. C. 214-222.

[6] Ebenfelt P. Normal forms and biholomorphic equivalence of real hypersurfaces in $\mathbb{C}^{3} / /$ Indiana Univ. Math. J. 1998. V. 47. № 2. P. 311-366.

[7] Владимиров В. С., Сергеев А. Г. Комплексный анализ в трубе будущего // Современные проблемы математики. Фундаментальные направления. Итоги науки и техники. Т. 8. М.: ВИНИТИ АН СССР, 1985. С. 191-266.

[8] Лобода А. В. Однородные вещественные гиперповерхности в $\mathbb{C}^{3}$ с двумерными группами изотропии // Тр. МИАН. 2001. Т. 235. С. 114-142.

Московский государственный университет им. М.В. Ломоносова

Поступило

E-mail: vkb@strogino.ru

05.07 .2004

Исправленный вариант

10.12.2004 